\title{
STRESS-STRAIN CURVE AND STORED ENERGY DURING UNIAXIAL DEFORMATION OF POLYCRYSTALS
}

\author{
W. Oliferuk, M. Maj \\ Institute of Fundamental Technological Research, Warsaw, Poland
}

\section{Introduction}

A portion of the mechanical energy expended on plastic deformation is released as a heat and the remainder is stored in the material. The stored energy is an essential feature of cold worked state and represents the change in the internal energy of the material.

The measurement of the stored energy is usually laborious and complicated therefore many authors have tried to calculate the stored energy from stress-strain curve though the curve does not contain information about the energy converted into a heat [1-3]. On the other hand both the strain hardening and stored energy of cold work are associated with the creation of lattice imperfections. Thus an attempt to find connection between stored energy and stress-strain curve seems to be justified.

The aim of this work is to answer the question what information about the stored energy can be derived from stress-strain curve. Results of theoretical study will be interpreted in terms of energy storage mechanisms and will be compared with stored energy determined experimentally during uniaxial tension.

\section{Energy balance during deformation}

The theoretical analysis of energy balance for elastic-perfectly plastic material has been performed. The curve shown in Fig. 1 is typical for 'load-unload cycle' for elastic-perfectly plastic material subjected to the load $F$.

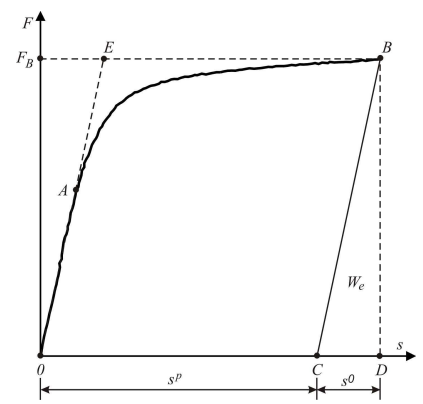

Fig. 1. Generalized load versus generalized displacement curve.

It has been shown that in the case of elastic-perfectly plastic material the total stored energy is equal to:

$$
E_{s}=W_{A E B}-\int_{V}^{\sigma_{B}} \int_{0}^{i p} \hat{\varepsilon}^{i p}: d \hat{\sigma} d V,
$$

where $\hat{\varepsilon}^{i p}$ is the local ideal plastic strain, what means that the total energy expended on this strain is converted into a heat, $\hat{\sigma}$ is the local stress tensor and $V$ is the volume of the gauge part of the specimen. 
It should be noticed that the energy $W_{A E B}$ can be calculated directly from experimentally obtained stress-strain curve (area $A E B$ in Fig. 1).

It has been shown that the energy $W_{A E B}$ is connected with internal stress field generated during nonhomogeneous plastic deformation. Performed analysis has shown that, on the basis of the stressstrain curve, it is impossible to derive the energy stored during homogeneous deformation (the energy of statistically stored dislocations).

\section{Experimental results}

The stored energy $e_{s}$ was determined as a difference between the mechanical energy expended on plastic deformation $w_{p}$ and the energy dissipated as a heat $q_{d}$

$$
e_{s}=w_{p}-q_{d},
$$

where $e_{s}, w_{p}, q_{d}$ are specific quantities.

The plastic work was determined on the basis of stress strain-curve. The energy $q_{d}$ was determined by simulating the process of specimen heating during deformation by means of controlled supply of electrical power in such a way that the temperature increase with time during the simulation was identical to that measured during the tensile test [4]. The temperature distribution on the specimen surface was determined using IR thermographic system.
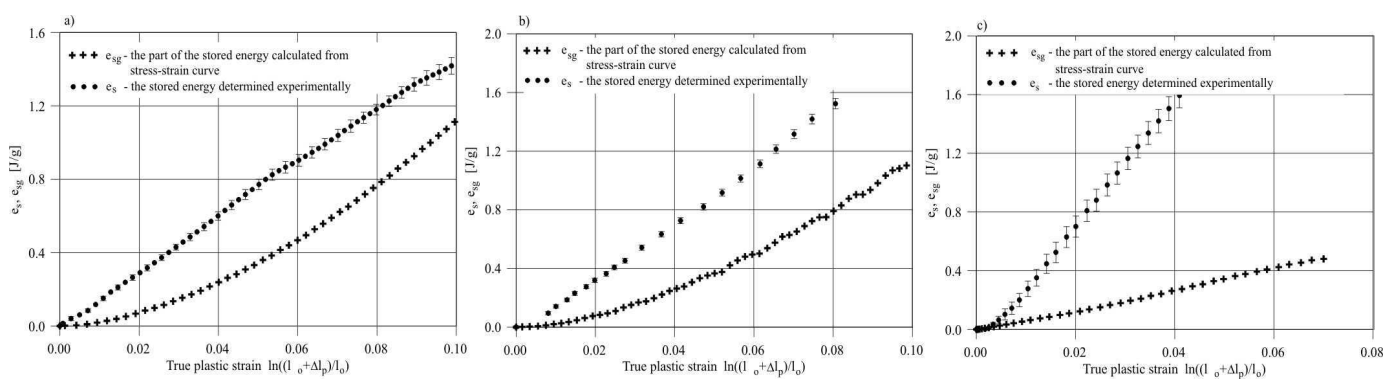

Fig. 2. The part of stored energy calculated from stress-strain curve and the measured total stored energy versus plastic strain for: a) 316L, b) 304L and c) Ti.

The results of total stored energy measurements performed on the $316 \mathrm{~L}$ and $304 \mathrm{~L}$ austenitic stainless steels and titanium, were compared with that obtained on the basis the theoretical analysis (Fig. 2). It is shown that the stored energy, connected with non-homogeneous plastic deformation, calculated from stress-strain curve for all tested materials is smaller than the total stored energy determined experimentally.

\section{References}

[1] V. Kafka (1979). Strain hardening and stored energy. Acta Tech. CSAV, 24, 199-216.

[2] N. Aravas, K.S. Kim, F.A. Leckie (1990). On the calculation of the stored energy of cold work. J. Eng. Mater. Techn., 112, 465-470.

[3] W. Szczepiński (2001). The stored energy in metals and the concept of residual microstresses in plasticity. Arch. Mech., 53, 615-629.

[4] W. Oliferuk, A. Korbel, M.W. Grabski (1996). Mode of deformation and the rate of energy storage during uniaxial tensile deformation of austenitic steel. Mater. Sci. Eng. A220, 123128. 